\title{
Stress and Self-Control as Predictors of Mobile Game Addiction Among Chinese University Students
}

\author{
Xiao Han ${ }^{1, a, *}$, Mengjun Li ${ }^{1, b}$, Ziqi Jiang ${ }^{1, c}$ and Zhouzhou Zhou ${ }^{1, d}$ \\ ${ }^{1}$ School of Journalism and Communication, Chinese University of Hong Kong, Hong Kong, \\ China \\ ahanxiao0810@126.com, rancylmj@link.cuhk.edu.hk, ${ }^{\mathrm{c}} 1155097148 @$ link.cuhk.edu.hk, \\ d1155097106@link.cuhk.edu.hk \\ *corresponding author
}

Keywords: Mobile Game Addiction, Stress, Self-Control

\begin{abstract}
The main purpose of this study is to discuss the behaviors of using mobile games and the causes of mobile game addiction among Chinese university students. Based on uses and gratifications theory, stress and self-control, these three aspects were used to analyze the motivations of mobile games use among university students. The study used a multi-stage sampling method and a sample of Chinese university students from eight departments of four universities in Beijing, Shanghai, Xi'an and Jinan was selected. 907 questionnaires were distributed and the number of valid questionnaires was 416 . The results show that social pressure and academic pressure are positively related to mobile game addiction, self-control ability is negatively related to mobile game addiction.
\end{abstract}

\section{大學生群體壓力、自控與手機遊戲成痕}

\section{韓䈗 $^{1, \mathrm{a}, *}$, 李夢珺 ${ }^{1, \mathrm{~b}}$, 姜子祺 ${ }^{1, \mathrm{c}}$, 周洲舟 ${ }^{1, \mathrm{~d}}$ \\ 1 新聞與傳播學院, 香港中文大學, 香港, 中國}

ahanxiao0810@126.com, brancylmj@link.cuhk.edu.hk, '1155097148@link.cuhk.edu.hk,

d155097106@1ink.cuhk.edu.hk

*通訊作者

關鍵字: 手機遊戲成瘧, 壓力, 自控能力

摘要: 本研究的主要目的, 是探討大學生群體使用手機遊戲行為及成癔的原因。 研究主要從「使用與滿足」、「壓力」、自控行為」三個層面來分析大學生群體對 手機遊戲使用的動因及其使用滿足感。本研究採用多階段抽樣法, 從中國大陸: 北京、上海、西安、濟南這四座城市的四所大學八個院系中隨機發放 907 份問卷, 回收有效問卷 416 份。通過對問卷的相關分析, 研究結果發現, 社交壓力、學業

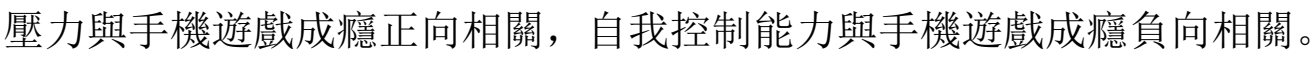

\section{1. 研究動機與目的}

智能科技的持續發展日漸豐富人們的娛樂方式，遊戲產業的持續化發展，使 得遊戲的呈現方式不再局限於電腦終端。手機遊戲的種類開始由單一的益智類單 
機遊戲向競技類網路遊戲偏移, 據[31]iiMedia Research 發佈的《2017 上半年中 國手機遊戲市場研究報告》顯示, 2016 年中國手遊用戶規模達 5.23 億人, 其中, 19-25 歲的年輕用戶是目前手遊活躍用戶的主力軍。

遊戲的過度使用所導致的成痕現象正影響著大學生群體的日常生活和學習， 並造成大學生群體對手機遊戲產生強烈持續的依賴感。大學校園中衍生出的手機 使用及管理問題，已經成爲高校教育管理部門與老師不得不正視與處理的重要課 題。目前, 雖然影響成癬行為因素的相關研究眾多, 但多數將研究目光聚焦於傳 播理論與遊戲使用與滿足方面。因此, 將行為因素（自控）與傳播理論（使用與 滿足) 相結合來研究手機遊戲成瘾具有重要的理論意義和現實意義。本研究旨在 通過利用問卷考察大學生群體手機遊戲使用行爲相關指標, 分析相關影響因素, 從而為預防和干預大學生手機遊戲成瘾相關措施提供依據和參考。

基於以上研究背景與動機, 本研究主要選取中國大陸的四個城市: 北京、上 海、西安、濟南，並從這四個城市的大學中抽取四個學院八個學系，對以下問題 進行了探討:

(1)探討大學生手機遊戲成痕玩家所喜愛的遊戲類型。

(2)探討社交壓力與學業壓力是否影響大學生群體手機遊戲成瘾。

(3)探討自控能力是否影響大學生群體手機遊戲成瘧。

\section{2. 文獻綜述}

\section{1 壓力與成痧}

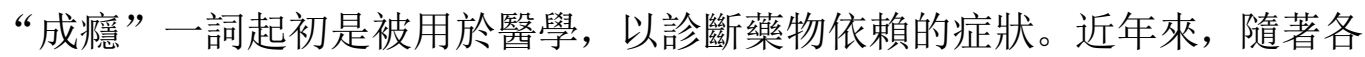
種社會問題以及群體現象的湧現, 有諸多學者將 “行為成瘤”用於評斷諸如 “賭 博” ([7]Alexander, 2017)、“過度縱欲” ([19]Rory, 2016)等許多行為現象。作為

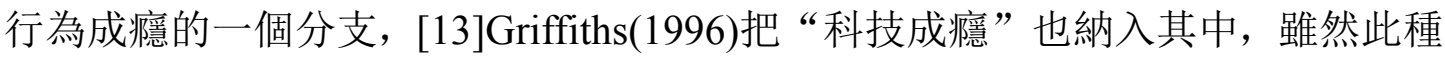
行為並非是藥物造成的依賴行為, 但是因為用戶的行為在與機器的互動中所呈現 的特徵與成瘜症狀非常類似, 故也稱為行為上的沉迷。[14]Hussai 等(2012)把無 法控制使用遊戲, 在停止使用遊戲後, 心理及生理上會出現一些反應的行為稱為 遊戲成癬。[29]Young(2009)則進一步將成瘧症狀分為著迷程度、耐受力、無法有 效控制及限制遊戲使用行為、心理戒斷和逃避現實等幾個方面。

近年來隨著互聯網的高速發展，人們開始借助網路來舒緩自身的壓力。有研 究者認為, 網路是人們用來暫時逃避問題的手段, 人們通過網路來擺脫因各種壓

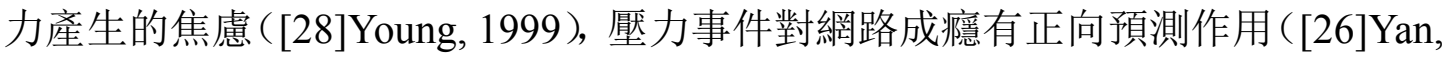
2014）。Yan 等人根據陳氏網路成瘧量表將結果分為: 非成瘧者、輕度網路成瘧 者及重度網路成瘧者, 然後通過青少年自我評價生活事件清單對壓力生活事件進 行了測量。回歸分析結果顯示精神過敏可能與生活壓力相互作用, 從而影響網路

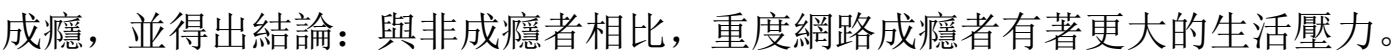

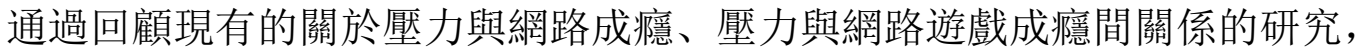
可以發現大學生涯力主要來自於學習、社會和個人方面, 有研究表明, 大學生最 大的壓力源是學業壓力, 其次是與社會環境相關的因素（[17]Misra, 2000）。

基於以上研究回顧, 本研究提出以下假設:

$\mathrm{H}_{1}$ ：社交壓力感越大的人，越容易對手機遊戲成瘧。

$\mathrm{H}_{2}$ ：學業壓力感越大的人，越容易對手機遊戲成瘧。

\section{2 自我控制能力與手機遊戲成瘜}

許多學者通過量化研究的方式對自我控制能力和成瘾之間的關係進行研究。 
[15]Kim 等人（2008）探討了網路遊戲成瘾與攻擊性、自我控制和自戀等心理特 徵之間的關係。他們選取韓國三個地區的 1471 名線上遊戲用戶進行問卷調查, 並對玩家的自我控制能力進行了測量, 研究結果證明了自我控制與網路遊戲成癃 分數成負相關的假設。而在這項研究中, 自我控制被定義爲 “延遲滿足”, 玩家

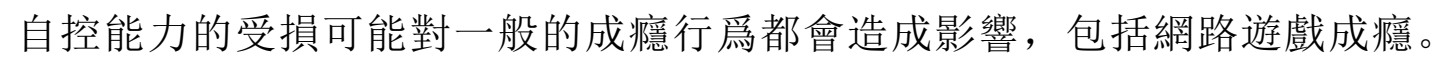

[9]Chen 和 Leung (2016) 通過發放網上問卷的形式 對中國 409 名玩 Candy Crush Saga 遊戲的受訪者進行了調查, 他們採用[23]Tangney 等人在 2004 年開發的自我 控制量表（BSCS）來測量一個人控制自己的能力。結果顯示，自我控制是移動

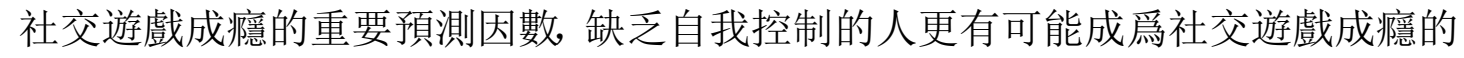
受害者, 針對這些缺乏自我控制的玩家, 研究預測嚴格的規章制度可能會有效地 阻止他們過度沉迷。值得注意的是, [11]Gabbiadini 等人(2014)在研究中經過對 172 名高中生進行道德脫離對比實驗, 結果發現暴力遊戲玩家的自我控制能力更 低。即表明暴力電子遊戲的確減少了人們的自我控制能力。

綜上所述, 基於已有研究, 本研究提出以下假設:

$\mathrm{H}_{3}$ ：自我控制能力差的人, 越容易對手機遊戲成痕。

\section{3 使用與滿足理論}

使用與滿足理論起源於二十世紀三四十年代, 該理論從媒介使用者的視角進 行探討, 並假設閱聽者會積極 主動地使用媒介, 以滿足他們的特定需求和需要。 因此，閱聽人是積極的、而非被動接受訊息的媒介使用者。([6]羅文輝、李秕青、 施盈廷、楊秀娟, 2015)。早前的研究大多圍繞廣播與讀者的關係展開, 隨著報 紙、電視等媒介的出現, 伴隨網路的迅速發展及新興媒介的湧現, 使用與滿足理 論不僅被運用於探討媒介使用者的新興媒介使用行為和動機([21]Ruggiero, 2000), 而且被認為該研究模型的研究取向對與新傳播媒介和互動式媒介的研究尤為重 要([20]Rubin, 2002)。隨著遊戲產業的出現與發展, 手機遊戲作為一種新型、體 驗式為導向的新媒介，同樣滿足了該媒介使用者各種各樣的目的。

[25]Wu等人(2010)從社交需求、愉悅感和成就感這三個方面探討了遊戲的使 用與滿足感。結果顯示在遊戲世界中, 使用者可以與之前已經認識的朋友保持互 動與聯絡, 也可以通過遊戲交到其他的新朋友; 成就排名、對遊戲角色的知悉度、 個人遊戲水準和遊戲虛擬物品擁有程度都會促進遊戲玩家的成就感。[10]Engl等 人(2013)則將手機遊戲作為一種消磨時間的方式，也有學者將逃避現實([27]Yee， 2006)和尋求放鬆([9]Chen \& Leung, 2016)作為手機遊戲玩家常見的滿足需求。

多數論文通常對遊戲使用的動機和原因([24]Wei \& Lu, 2014; [10]Engl \& Nacke, 2013)進行研究, 試圖分析出不同種類的特定遊戲玩家的社會屬性或心理 特性, 以及遊戲需求是如何影響他們的遊戲動機。探討遊戲使用者的滿足需求與 遊戲成瘜程度之間的關係([30]Zhou \& Leung, 2012), 以及滿足需求是如何與手機

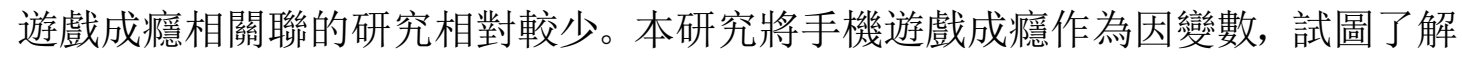
大學生遊戲使用者在社交、逃避現實、放鬆、成就感、娛樂這五個方面的滿足需 求與手機遊戲成癔度之間的關係。同時, 本研究也力圖探討大學生群體手機遊戲 的使用情況和使用行為是如何滿足他們的需求動機, 以及何種需求最容易引起手 機遊戲成瘦。

\section{3. 研究方法}

\section{1 抽樣方法}

本研究採用多階段抽樣方法, 主要分析中國大陸大學生群體手機遊戲成瘜的 
相關影響因素。由於群體基數巨大、推斷面廣, 因此研究地域選取手遊用戶聚集 地: 北京、上海、西安、濟南四個城市, 這在一定程度上代表了手遊人群的集體 特性。研究將北京、上海、西安、濟南的所有一本大學編號, 從中隨機抽取一所 高校, 再從這一所高校中隨機抽取兩個學院 在本科和碩士兩個階段所在年級中, 以相同比例, 隨機抽取發放問卷。北京抽取的是北京師範大學, 上海抽取的是上 海電影學院，西安抽取的是西北大學，濟南抽取的是山東大學。問卷發放時間為 2017 年 11 月初至 2017 年 11 月中旬, 通過網上問卷方法的方式共發放問卷 905 份，有效問卷為 416 份，有效發放問卷率為 $46.0 \%$ 。

\section{2 研究變項}

（一）使用與滿足價值

本研究測量受訪者的手機遊戲使用動機的方法, 是參考[27]Yee(2006)的研究, 依照使用與滿足理論把使用與滿足價值分為五個方面: 社交、逃避現實、放鬆、 成就感和娛樂。用 20 個題分別詢問受訪者手機遊戲使用是否可以滿足他們的需 要。研究分別把其他題項受訪者使用與滿足的不同動機題目相加除以二，構成 “社交”指標（ $\mathrm{M}=3.37, \mathrm{SD}=0.87)$ 、“逃避現實”指標（ $\mathrm{M}=2.77, \mathrm{SD}=1.05)$ 、 “放鬆” 指標（ $\mathrm{M}=3.69, \mathrm{SD}=0.86) 、 “$ 成就感” 指標 $(\mathrm{M}=3.23, \mathrm{SD}=0.90)$ 和 “娛樂”指標（M=2.79，SD=1.12）這五個方面。

表 1 :使用與滿足價值分析

\begin{tabular}{|c|c|c|c|}
\hline & 指標 & 平均數 & 標準差 \\
\hline \multirow{2}{*}{ 社交 } & 通過手機遊戲我能和朋友增進感情 & 3.63 & 0.95 \\
\hline & 手機遊戲使我交到了新的朋友 & 3.10 & 1.04 \\
\hline \multirow{2}{*}{$\begin{array}{c}\text { 逃避现 } \\
\text { 实 }\end{array}$} & 玩遊戲時我忘記了現實生活中存在的一些問題 & 2.71 & 1.22 \\
\hline & 我在遊戲中可以投入自我忘記現實環境 & 3.58 & 1.01 \\
\hline \multirow{2}{*}{ 放松 } & 玩遊戲是一個很好的發洩方式 & 3.79 & 0.99 \\
\hline & 玩遊戲可以很好的消磨時間 & 2.82 & 1.17 \\
\hline \multirow{2}{*}{ 成就感 } & 我在意自己在好友圈中的遊戲排名 & 2.61 & 1.23 \\
\hline & 我解決有挑戰性的關卡後感到很有成就感 & 3.85 & 1.00 \\
\hline 娱乐 & 我很享受沉浸於遊戲世界中 & 2.79 & 1.12 \\
\hline
\end{tabular}

測量 “社交壓力” 的方法, 參考[3]劉路(2015)的相關研究, 從外形、與同學 相處情況 社會適應能力、經濟狀況四個層面共四個題項測量受訪者的社交壓力。 在進行統計分析時, 我們把受訪者在這 4 個題項的得分相加, 組成受訪者的 “社 交壓力”指標 $(\mathrm{M}=11.52, \mathrm{SD}=3.58)$ 。

（三）學業壓力

本研究測量學業壓力的方法, 是參考過去的研究文獻[1]李虹(2002), 從考試 考證情況、作業數量、成績排名三個層面共三個題項測量受訪者的學業壓力。建 構學業壓力的方法, 是把這三個題目的得分相加, 得到 學業壓力」指標 $(\mathrm{M}=9.07$, $\mathrm{SD}=2.07)$ 。

(四) 自控能力

測量受訪者的自我控制能力的方法, 是參考[23]Tangney(2004)和[4]譚樹華等 (2008)的研究, 並此基礎上進行了改編, 分別用 9 個題測量受訪者的自我控制能 力高低，在分析時把這九個題加起來，構成 “自控能力” 指標 
$(\mathrm{M}=33.37, \mathrm{SD}=4.96)$ 。

(五) 手機遊戲成癌

手機遊戲成瘧的測量, 是主要參考[2]陳淑惠(2003)和鍾才元(2013)所發展的 題項, 用 12 個題目 (行為、心理) 測量受訪者的手機遊戲成瘧程度。在分析時, 把這 12 個題項得分相加後除以 12 構成 “手機遊戲成瘜” 指標 $(\mathrm{M}=2.39, \mathrm{SD}=0.77)$ 。

\section{（六）人口變項}

本研究採用的控制變項有: 性別、年齡、就讀年級, 上學期平均學業成績滿 意度，這些變項在相關分析中當成控制變項。

\section{4. 資料分析}

在本研究的 416 位受訪者中，男性占 $54.1 \%$ 女性占 $45.9 \%$ 平均年齡為 19.91 歲。在上學期學業成績滿意度方面, 非常不好的占 $6.3 \%$, 中等占 $21.4 \%$, 非常 好占 $3.4 \%$, 有些不好占 $20.2 \%$, 還算好 $27.4 \%$, 不知道占 $21.4 \%$ 。在每週玩手機 遊戲的天數中, 每天都玩的占 $53.6 \%$, 六天都玩的占 $1.7 \%$, 五天都玩的占 $6.5 \%$, 四天的占 $4.3 \%$, 三天的占 $10.6 \%$, 兩天的占 $8.7 \%$, 一天的占 $3.6 \%$, 幾乎不玩的 占 $11.1 \%$ 。而在每天玩手機遊戲的時間長度中， 15 分鐘至 30 分鐘的占 $22.1 \%$, 30 分鐘以上至 1 小時的占 $22.6 \% ， 1$ 小時至 2 小時占 $20.7 \%$, 而 6 小時以上則只 占 $1.9 \%$ 。在最常玩的遊戲種類中, 最受歡迎的休閒益智類占 $48.3 \%$, 競技類占 $37.7 \%$ ，角色扮演類占 $29.3 \%$ ，最不受歡迎的射擊類只占 $8.9 \%$ 。

為驗證本研究的三個假設, 驗證方法採用相關分析。表二表示相關分析的結 果, 控制變數包括性別、年齡、就讀年級、上學期平均學業成績滿意度, 因變項 為「手機遊戲成痉」。

表 2: 人口變項, 使用與滿足變項, 社交壓力變項, 學業壓力變項, 自控能力變項 和手機遊戲成痉相關分析

\begin{tabular}{ccc}
\hline & 預測變項 & 手機遊戲成瘾 \\
\hline \multirow{2}{*}{ 人口變項 } & 性別 & $-.100^{*}$ \\
& 年齡 & .024 \\
& 就讀年級 & .055 \\
& 上學期平均學業成績滿意度 & $-.116^{*}$ \\
\hline 使用與滿足變項 & 社交 & $.175^{* * *}$ \\
& 逃避現實 & $.387^{* * *}$ \\
& 放鬆 & $.255^{* * *}$ \\
& 成就感 & $.318^{* * *}$ \\
& 娛樂 & $.384^{* * *}$ \\
\hline & 社交壓力 & $.421^{* * *}$ \\
學業壓力 & $.331^{* * *}$ \\
自控能力 & $-.221^{* * *}$
\end{tabular}

1): 雙尾檢驗相關係數

2)：變項編碼方式：性別（1=男；2=女); 就讀年紀 ( $1=$ 本科一年級; $5=$ 研 究生); 上學期平均學業成績滿意度 ( $1=$ 非常不好; $5=$ 非常好; $6=$ 不知道); 社 交, 逃避現實, 放鬆, 成就感, 娛樂 (1=非常同意; 5=非常不同意); 社交壓力, 學業壓力, 自控能力, 手機遊戲成㾤 ( 1 =非常符合; $5=$ 非常不符合); $\mathrm{N}=416$ (資 
料分析包括全部的有效受訪者)。

3): ${ }^{*} \mathrm{p}<.05,{ }^{* *} \mathrm{p}<.01,{ }^{* * *} \mathrm{p}<.001$

分析結果發現，性別（ $\mathrm{r}=-.100, \mathrm{p}<.05 ）$ 和上學期平均學業成績滿意度 $(\mathrm{r}=-.116, \mathrm{p}<.05)$ 均能顯著預測手機遊戲成瘾。在使用與滿足方面, 逃避現實是最 有利的預測變項 $(\mathrm{r}=.387, \mathrm{p}<.001)$ ，其次是娛 樂 $(\mathrm{r}=.384, \mathrm{p}<.001)$ 、成就感 $(\mathrm{r}=.318, \mathrm{p}<.001)$ 、放鬆（ $\mathrm{r}=.255, \mathrm{p}<.001 ） 、$ 社交 $(\mathrm{r}=.175, \mathrm{p}<.001)$, 這五個變項均能顯

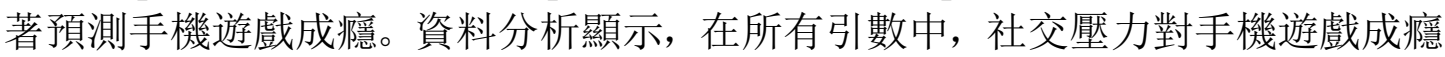
有最顯著的正相關預測力 $(r=.421, \mathrm{p}<.001)$, 其次是學業壓力 $(\mathrm{r}=.331, \mathrm{p}<.001)$, 自控能力對手機遊戲成痑有顯著的負相關預測力（ $\mathrm{r}=-.221, \mathrm{p}<.001 ） 。$ 因此，本研 究三個假設獲得支持。

\section{5. 結論與建議}

研究結果表明, 社會現實壓力可以顯著預測手機遊戲的成疻趨勢, 即大學生 群體對手機成瘧的主要動因是環境壓力因素, 而手機遊戲能夠提供的逃避空間所 導致的大學生逃避行為, 成為大學生群體持續手機遊戲沈溺的主要原因。學業或 社交壓力感越大的同學, 越容易對手機遊戲成癋, 這一研究結果與過去其他學者 的研究相一致 ([16]Lee \& Shin, 2004)。在使用與滿足方面, 逃避現實的需求是 最顯著的預測變項, 遊戲的娛樂性和從遊戲任務設置環節中所獲得的成就感同時 也是預測大學生選擇手機遊戲種類並是否容易成瘦的有利變項。在自控方面, 研 究發現自控能力對手機遊戲的成痞有著非常顯著的預測能力, 即個人的自控能越

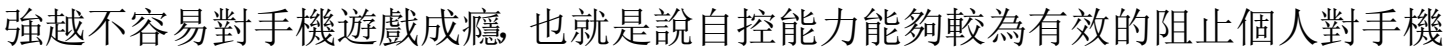
遊戲的長時間沈溺參與。根據上述研究發現, 在環境和學業的雙重壓力下, 大學 生群體比較容易產生使用手機遊戲進行解壓的逃避行為,持續這種行為而無自控 能力的約束就容易形成對手機遊戲成瘾無法自拔。

在過去對遊戲成癣的相關研究中，我們發現，研究者傾向於將使用與滿足理 論和自控能力分開研究, 成為兩種不同的研究方向, 討論從期望值到使用之滿足 兩個變項對成瘧行為的影響，或自控能力在遏止成瘧行為中的作用等。與過去的 相關研究相比, 本研究將自控能力作為重要的變項考慮, 並與使用與滿足理論相 互作用，綜合考察了大學生群體手機遊戲成癋的相關因素。因此，在大學生手機 遊戲成瘤的社會問題上, 本研究有著重要的現實意義。參照本研究的結果, 探詢 如何遏止大學生長時間沈溺手機遊戲的措施,應將重點放在培養提高學生的自控 能力上，這是有效的防止大學生手機遊戲成癬行為發生的重要途徑。

\section{参考文献}

[1] 李虹,梅錦榮（2002）.〈大學生壓力量表的編制〉.《應用心理學》,第 8 卷第 1 期,頁 $27-32$.

[2] 陳淑惠,翁儷禎,蘇逸人（2003）。〈中文網路成癤量表之編制與心理計量特性 研究〉.《中華心理學刊》,第 3 期,頁 $279-282$.

[3] 劉路（2015）。〈研究生學習壓力量表的應用及其信效度分析〉。《克拉瑪依學 刊》,2015 年第 1 期,頁 $71-74$.

[4] 譚樹華, 郭永玉（2008）。〈大學生自我控制量表的修訂〉。《中國臨床心理學 雜誌》,第 16 卷第 5 期,頁 $468-470$.

[5] 鐘才元,陳明終,賴阿福,楊政穎,林慧婷（2013）。〈網路與手機雙重成痕者之人 格特質分析〉,「TANET2013 臺灣網際網路研討會」論文,臺灣. 
[6] 羅文輝,李鬱青,施盈廷,楊秀娟（2005）。〈網路採用,使用與獲得之滿足〉。《新 聞學研究》,第 83 期,頁 $127-165$.

[7]Alexander, T., Jacques, B., \& Jeremy, J. (2017). Linking empathy to visuospatial perspective-taking in gambling addiction. Psychiatry Research, 250, 177-184.

[8]Baumeister, R. F. (2003). Ego Depletion and Self-Regulation Failure: A Resource Model of Self-Control. Alcoholism: Clinical and Experimental Research, 27(2), 281-284.

[9]Chen, C., \& Leung, L. J. (2016). Are you addicted to Candy Crush Saga? An exploratory study linking psychological factors to mobile social game addiction. Telematics and Informatics, 33, 1155-1166.

[10]Engl, S., \& Nacke, L. E. (2013). Contextual influences on mobile player experience - A game user experience model. Entertainment Computing, 4 (1), 83-91.

[11]Gabbiadini, A., Riva, P., Andrighetto, L., Volpato, C., \& Bushman, B. J. (2014). Interactive Effect of Moral Disengagement and Violent Video Games on Self-Control, Cheating, and Aggression. Social Psychological and Personality Science, 5(4), 451-458.

[12]Goeders, N. E. (2003). The impact of stress on addiction. European Neuropsycharmacology, 13(6), 435-441.

[13]Griffiths, M. (1996). Gambling on the internet: A brief note. Journal of Gambling Studies, 12(4), 471-473.

[14]Hussain, Z., Griffiths, M.D., \& Baguley, T. (2012). Online gaming addiction: classification, prediction, and associated risk factors. Addiction Research \& Theory, 20(5), 359-371.

[15]Kim, E. J., Namkoong, K., Ku, T.\& Kim, S. J. (2008). The relationship between online game addiction and aggression, self-control and narcissistic personality traits. European Psychiatry, 23(3), 212-218.

[16] Lee, O., \& Shin, M. (2004). Addictive consumption of avatars in cyberspace. CyberPsychology and Behavior, 7(4), 417-420.

[17]Misra, R., \& McKean, M. (2000). College students academic stress and its relmion to their anxiety, time management and leisure satisfaction. American Journal of Health Studies, 16(1), 41-51.

[18]Myrseth, K.O.R., \& Fishbach, A. (2009). Self-Control A Function of Knowing When and How to Exercise Restraint. Association for Psychological Science, $18(4), 247-252$.

[19]Rory, C. R. (2016). Additional challenges and issues in classifying compulsive sexual behavior as an addiction. Addiction, 111(12), 2111-2113.

[20]Rubin, A. M. (2002). The uses-and-gratifications perspective of media effects. Media effects: Advances in theory and research, 525-548.

[21]Ruggiero, T.E. (2000). Uses and gratifications theory in the 21st Century. Mass Communication and Society, 3(1), 3-37.

[22]Scharkow, M., Festl, R., Vogelgesang, J., \& Quandt, T. (2015). Beyond the "core-gamer": Genre preferences and gratifications in computer games. Computers in Human Behavior, 44, 293-298.

[23]Tangney, J.P., Baumeister, R.F., \& Boone, A.L. (2004). High Self-Control Predicts Good Adjustment, Less Pathology, Better Grades, and Interpersonal Success. Journal of Personality, 72(2), 271-322.

[24]Wei, P. S., \& Lu, H. P. (2014). Why do people play mobile social games? An examination of network externalities and of uses and gratifications. Internet Research, 24(3), 313-331. 
[25]Wu, J.H., Wang, S.C., \& Tsai, H.H. (2010). Falling in love with online games: the uses and gratifications perspective. Computers in Human Behavior, 26(6), $1862-1871$.

[26]Yan, W., Li, Y.H. \& Sui, N. (2014). The Relationship between Recent Stressful Life Events, Personality Traits, Perceived Family Functioning and Internet Addiction among College Students. STRESS AND HEALTH, 30(1), 3-11.

[27]Yee, N. (2006). The Demographics, Motivations, and Derived Experiences of Users of Massively Multi-User Online Graphical Environments. Teleoperators and Virtual Environments, 15(3), 309-329.

[28]Young, K.S. (1999). Internet addiction: Symptoms, evaluation and treatment. Clinical Practice, 17, 19-31.

[29] Young, K.S. (2009). Understanding online gaming addiction and treatment issues for adolescents. American Journal of Family Therapy, 37(5), 355-372.

[30]Zhou, S., \& Leung, L. (2012). Gratifications, loneliness, leisure boredom, and self-esteem as predictors of SNS-game addiction and usage pattern among Chinese college students. International Journal of Cyber Behavior, Psychology and Learning, 2(4), 34-48.

[31]艾媒諮詢（2017年8月25日）。〈2017上半年中國手機遊戲市場研究報告〉。 取自http://www.iimedia.cn/54970.html 